\title{
Mechanisms of Response to Salt Stress in Oleander (Nerium oleander L.)
}

\author{
Dinesh KUMAR ${ }^{1,2}$, Mohamad AL HASSAN², Oscar VICENTE², Veena AGRAWAL ${ }^{1}$, Monica BOSCAIU ${ }^{*}$ \\ ${ }^{1}$ Department of Botany, University of Delhi, India \\ ${ }^{2}$ Instituto de Biología Molecular y Celular de Plantas (UPV-CSIC) and ${ }^{3}$ Instituto Agroforestal \\ Mediterráneo (UPV), Universitat Politècnica de València, Spain \\ *)Corresponding author, e-mail: mobosnea@eaf.upv.es
}

BulletinUASVM Horticulture 73(2) / 2016

Print ISSN 1843-5254, Electronic ISSN 1843-5394

DOI:10.15835/buasvmcn-hort:12287

\begin{abstract}
Elucidating the mechanisms of abiotic stress tolerance in plants will help to develop more resistant crop varieties, improving agricultural production in a climate change scenario. Basic responses to salt stress (osmolyte accumulation, activation of antioxidant systems), have been studied in Nerium oleander, a xerophytic species widely used as ornamental. Salt strongly inhibited growth, but the plants survived one month in the presence of 800 $\mathrm{mM} \mathrm{NaCl}$, indicating that the species is also relatively resistant to salt stress. Salt induced a slight increase in the levels of proline, glycine betaine and soluble sugars; the latter compounds showed much higher absolute contents, suggesting a functional role in osmotic adjustment, and the presence of constitutive mechanisms of response to salt stress. High salinity generated oxidative stress in the plants, as shown by the increase of malondialdehyde levels. Antioxidant systems, enzymatic and non-enzymatic, are generally activated in response to salt stress; in oleander, they do not seem to include total phenolics or flavonoids, antioxidant compounds which did not accumulate significantly in salt-treated plants.
\end{abstract}

Keywords: climate change; Nerium oleander; salt stress; soil salinisation

\section{Introduction}

Soil salinity is one of the most important causes of reduction of crop yields worldwide, an increasing problem due to climate change. Genetic improvement of salt tolerance of our crops has become an urgent need for the future of agriculture, especially in arid and semiarid regions (Fita et al., 2015). Although all plants activate the same basic responses to salt stress (control of ion transport, osmotic adjustment, activation of antioxidant systems, etc.), the relative relevance of these conserved responses for the tolerance of a given species is largely unkown. Elucidating the most efficient mechanisms of tolerance in different plants will contribute to increasing agricultural production and to global food security.

\section{Aims and objectives}

This work reports an initial characterisation of the responses to salt stress in the Mediterranean shrub Nerium oleander, a xerophyte (droughttolerant plant) widely used as ornamental, which has been also described as moderately resistant to salinity.

\section{Materials and methods}

One-year-old $N$. oleander plants were treated for one month with 400 and $800 \mathrm{mM} \mathrm{NaCl}$ in half-strength Hoagland nutritive solution, while control plants were grown in parallel in the absence of salt. After harvesting, several growth parameters were measured, and the contents of common osmolytes [proline (Pro), glycine 
Tab. 1. Salt stress-induced growth inhibition in Nerium oleander. Growth parameters: fresh weight (FW), stem length (LS), in control and salt stressed plants, after one-month treatments with the indicated $\mathrm{NaCl}$ concentrations

\begin{tabular}{c|c|c}
\hline $\mathrm{NaCl}$ treatments $(\mathrm{mM})$ & FW $(\%)$ & SL $(\%)$ \\
\hline 0 (control) & 100 & 100 \\
\hline 400 & $30.7 \pm 6.8$ & $14.3 \pm 2.3$ \\
\hline 800 & $24.6 \pm 3.3$ & $10.0 \pm 2.5$ \\
\hline
\end{tabular}

Tab. 2. Relative changes in leaf levels of proline (Pro), glycine betaine (GB), total soluble sugars (TSS), malondialdehyde (MDA), total phenolic compounds (TPC) and total flavonoids (TF), in Nerium oleander plants, after one-month salt treatments with the indicated $\mathrm{NaCl}$ concentrations, as compared to non-stressed control plants (considered as 100\% for each measured compound)

\begin{tabular}{ccccccc}
\hline NaCl treatments (mM) & Pro (\%) & GB (\%) & TSS (\%) & MDA (\%) & TPC (\%) & TF (\%) \\
\hline 0 (control) & 100 & 100 & 100 & 100 & 100 & 100 \\
\hline 400 & $158 \pm 12$ & $170 \pm 6$ & $126 \pm 4$ & $157 \pm 15$ & $93 \pm 4$ & $104 \pm 7$ \\
\hline 800 & $125 \pm 12$ & $171 \pm 5$ & $126 \pm 4$ & $189 \pm 9$ & $113 \pm 5$ & $135 \pm 4$ \\
\hline
\end{tabular}

betaine (GB) and total soluble sugars (TSS)] and of some non-enzymatic antioxidants [total phenolic compounds (TPC), total flavonoids (TF)], as well as those of malondialdehyde (MDA, an oxidative stress marker), were determined using spectrophotometric assays.

\section{Results and discussion}

Salt inhibited growth of $N$. oleander in a concentration-dependent manner, as shown by a marked decrease of stem length and fresh weight, already observed in $400 \mathrm{mM} \mathrm{NaCl}$-treated plants and stronger at $800 \mathrm{mM} \mathrm{NaCl}$ (Tab. 1.). Nevertheless, the very fact that the plants survived for one month these high salinity conditions indicates that this species is relatively resistant to salt stress, not only to drought.

The relative levels of common osmolytes (Pro, GB and TSS), increased in plants treated with $400 \mathrm{mM} \mathrm{NaCl}$, as compared to the controls, but only slightly - by less than two-fold, in all cases - with the smallest variation observed in TSS. Increasing salt concentration to $800 \mathrm{mM}$ did not lead to higher osmolyte accumulation (Tab. 2.). Yet, absolute TSS contents were much higher than those of the other osmolytes, both in salt-treated and in control plants, reaching nearly $100 \mathrm{mg}$ eq. glucose $\mathrm{g}^{-1} \mathrm{DW}$. Therefore, soluble carbohydrates are probably the major functional osmolytes in oleander, accumulating at high concentrations even in the absence of stress; this suggests the presence of constitutive mechanisms of defence against abiotic stress, supporting the hypothesis of the 'pre-adaptation' to stress in salt-tolerant plants.

The salt treatments induced oxidative stress in oleander plants, as revealed by the relative increase in MDA levels (Tab. 2.). Changes in the contents of non-enzymatic antioxidants, such as TPC or TF, were however very small or not significant (Tab. 2.), indicating that other antioxidant systems, enzymatic and/or non-enzymatic, should be activated to counteract the salt-induced oxidative stress.

\section{Conclusions}

TSS appear to be the main contributors to osmotic adjustment in Nerium oleander, and their presence at high levels in non-stressed plants may represent a constitutive mechanism of response to salt stress. High external salt concentrations generate oxidative stress in oleander plants; antioxidant systems, which could be activated to counteract it, do not seem to include phenolic compounds or flavonoids.

Acknowledgements. D.K. was recipient of a scholarship from the NAMASTE programme (EU-India), which funded his stay at the IBMCP laboratory in Valencia. 


\section{REFERENCES}

1. Fita, A., Rodríguez-Burruezo, A., Boscaiu, M., Prohens, J. and Vicente, O. (2015). Breeding and domesticating crops adapted to drought and salinity: A new paradigm for increasing food production. Front Plant Sci 6:978. doi: 10.3389/fpls.2015.00978. 